\title{
Desigualdad en los Derechos Humanos de las mujeres mayores: caso Estado de Zacatecas
}

\section{Inequality in the Human Rights of older women: case of the State of Zacatecas}

\author{
Víctor-Hugo Bañuelos-García \\ Universidad Autónoma de Zacatecas. Unidad Académica de Contaduría \\ y Administración, Zacatecas, México \\ $\triangle$ victorhugo@unizacatecas.edu.mx \\ (iD) https://orcid.org/0000-0003-0888-4157
}

\section{Flor-de-María García-Martínez}

Universidad Autónoma de Zacatecas. Unidad Académica

de Psicología, Zacatecas, México

$\bowtie$ flordemaria@unizacatecas.edu.mx

(iD) https://orcid.org/0000-0003-3869-0169

\section{José-Rogelio Hernández-Ponce}

Universidad Autónoma de Zacatecas. Unidad Académica

de Contaduría, Zacatecas, México

$\triangle$ jrogelio hernandez@yahoo.com.mx

Recibido: 19-09-2019

D https://orcid.org/0000-0003-2015-6339

Aceptado: $12-12-2019$

\section{RESUMEN}

De acuerdo con la Organización Mundial de la Salud (OMS), 2015, entre 2000 y 2050, a nivel mundial, la población de adultos mayores de 60 años pasará del $11 \%$ al 22\%. En México, de acuerdo con el Consejo Nacional de Población (CONAPO), 2019, alcanzará cerca de 34\%. Referente al Estado de Zacatecas, México, se estima que irá del $11.08 \%$ en 2015 al $14.5 \%$ en 2030 . Este fenómeno significará un problema social que implica pobreza, discapacidad, abandono y enfermedades, entre otras condiciones, que, de no considerarse, se verán violentados los Derechos Humanos de sus habitantes. El objetivo del presente estudio consistió en evaluar y determinar la desigualdad de los Derechos Humanos que viven las mujeres mayores, en el Estado de Zacatecas, México, para lo cual se utilizó un cuestionario aplicado a 2.566 personas. Se hizo un análisis factorial exploratorio, en el que se encontraron tres factores: el primero de ellos denominado apoyo familiar, el segundo abuso físico y psicológico, el tercero, dependencia física y servicios. Además, mediante la Chi Cuadrada de Pearson, se encontraron relaciones estadísticamente significativas entre ser mujer y desigualdad económica, laboral y discapacidad. Por tanto, se concluye que, en el caso del Estado de Zacatecas, existe una transgresión de los Derechos Humanos de la Mujeres Mayores.

Palabras clave: Adultos mayores; derechos humanos; desigualdades; mujeres. 


\section{ABSTRACT}

According to the World Health Organization (WHO), 2015, between 2000 and 2050, worldwide, the population of adults over 60 will go from $11 \%$ to $22 \%$. In Mexic, according to the National Population Council (CONAPO), 2019, it will reach about 34\%. Regarding the State of Zacatecas, Mexico, it is estimated that it will go from $11.08 \%$ in 2015 to $14.5 \%$ in 2030 . This phenomenon will mean a social problem that implies poverty, disability, abandonment and diseases, among other conditions, which, if not considered, is the human rights of its inhabitants will be violated. The objective of this study was to evaluate and determine the inequality of Human Rights that older women live in, in the State of Zacatecas, Mexico, for which a questionnaire was used, applied to 2,566 people. An exploratory factor analysis was made, in which three factors were found: the first of them called family support, the second physical and psychological abuse, the third physical dependence and services. In addition, through Pearson's Chi Square, statistically significant relationships were found between being a woman and economic, labor and disability inequality. Therefore, it is concluded that, in the case of the State of Zacatecas, there is a violation of the Human Rights of Older Women.

Keywords: Older adults; human rights; inequalities; women.

\section{INTRODUCCIÓN}

Desde 1948, año en que fue proclamada la Declaración Universal de los Derechos Humanos, misma que expresa, los derechos y libertades a que somos meritorios los seres humanos, de manera exclusiva y en condiciones de igualdad. Por consiguiente, todos los seres humanos nacen libres e iguales en dignidad y derechos (Instituto Latinoamericano de las Naciones Unidas para la Prevención del Delito y Tratamiento del Delincuente (ILANUD), 2001). De esta declaración se deriva que todas las personas tienen derechos económicos, sociales, culturales y cívicos, entre otros, lo anterior sin importar la raza, sexo, clase, casta, creencia religiosa, edad u orientación sexual, motivo por lo cual se busca trabajar para lograr la igualdad y dignidad de todas las personas, según lo indica la (Comisión Nacional de Derechos Humanos (CNDH), 2018).

Sánchez (2018), señala que de acuerdo a la correspondencia con la mujer, en la Declaración Universal de Derechos Humanos se hace referencia expresa a ésta en dos circunstancias: en el preludio, en el que apunta a la igualdad de derechos de hombres y mujeres como uno de los miramientos que sustentan el texto, así como lo que corresponde al artículo 16 , en el que estipula la igualdad de hombres y mujeres frente al matrimonio. El manejo del lenguaje en el resto del contenido es impersonal, lo que da acceso a los derechos tanto a hombres como mujeres; sin embargo, hasta el momento de la admisión de la Declaración no existían instrumentos internacionales que respondieran a las necesidades específicas de la mujer. Hubieron que transcurrir 18 años precedentemente para que la Asamblea General acogiera tratados para la firma: Ios Pactos Internacionales.

Carrera (2019), cita que a partir de 1975, que se celebró en México la primer Conferencia Mundial sobre la Mujer y fue hasta junio del 2000, cuando se evaluó en Nueva York, la plataforma de trabajo de la Conferencia Mundial de Pekín, China, se llevaron a cabo una serie de labores y encuentros internacionales, en donde los gobiernos del mundo discuten sobre la problemática de la mujer y exhortan a realizar gestiones concretas con el fin de abatir la discriminación en diferentes aspectos de la vida de éstas. No obstante, la violencia contra las mujeres fue reconocida hasta hace muy poco, como una violación a sus derechos humanos. El sistema de derechos humanos no vislumbraba la violencia 
Bañuelos et al - Desigualdad en los Derechos Humanos de las mujeres mayores: Caso Estado de Zacatecas.

-aun la sexual como algo grave y significativo o digno de ser tomado en cuenta. La violencia contra las mujeres no podía ser enmarcada dentro del discurso de los derechos humanos. Por tal hecho, todos los tipos de violencia contra la mujer, la tortura y los eventos violentos que limitan su existencia concurrieron fuera del sistema de derechos humanos, lo que permitió la impunidad tanto a los generadores de violencia, como al Estado por excluir la creación de políticas públicas con el fin de advertir y condenar la violencia, así como espacios de atención a víctimas (Carrera, 2019), manifiesta que la campaña mundial de las mujeres pertenecientes a organizaciones no gubernamentales y de algunos gobiernos sensibles a este tema, crearon las circunstancias para alcanzar al primer reconocimiento característico de que la violencia contra la mujer es un tema de derechos humanos. La iniciativa logró concretarse durante la celebración de la Conferencia Mundial de Derechos Humanos celebrada en Austria, Viena, en 1993. Los documentos de Naciones Unidas posteriores a esta Conferencia, dieron cuenta de la búsqueda de una nueva terminología de género. En el año de 1998, la organización internacional que vela por el respeto a los derechos humanos, Amnistía Internacional, acogió oficialmente el uso de los términos derechos de la persona humana, derechos humanos, con lo que puso fin al uso del término derechos del hombre.

De acuerdo a la Organización de las Naciones Unidas (ONU), 2014, en su nota conceptual relativa al proyecto de recomendación general sobre la mujer de edad y la protección de sus Derechos Humanos, aprobó una recomendación sobre los Derechos Humanos de la mujer de la tercera edad, para evitar discriminación y tener condiciones de igualdad, dado que las mujeres tienden a vivir más que los hombres y esto genera que tienden a vivir solas. La recomendación se regirá de acuerdo con los principios de las Naciones Unidas en favor de personas de edad en el año de 1991, el Plan de Acción de Madrid sobre el Envejecimiento en el año 2002 y el Plan de Acción Internacional sobre el Envejecimiento en Viena, 1983. La mujer sufre de discriminación generada por estereotipos; principalmente, pertenecen a un sector pobre o sufren discapacidad, agravado por el hecho de que las mujeres de zonas rurales sufren tasas de analfabetismo que limitan acceso a la información de sus derechos; asimismo, de discriminación por empleos mal remunerados. En la Recomendación General No 25, sobre el Párrafo 1, del Artículo 4 de la Convención sobre la eliminación de todas las formas de discriminación contra la mujer, la Oficina del Alto Comisionado de las Naciones Unidas menciona que, a pesar de las resoluciones, declaraciones y recomendaciones aprobadas este organismo, las mujeres siguen siendo objeto de importantes exclusiones, violando con esto la igualdad de derechos, por lo que se condena la discriminación en todas sus formas. Asimismo, se busca se adecuen las legislaciones que constituyan discriminación hacia ellas.

Conforme al Informe Regional de Desarrollo Humano 2013-2014, la Organizacion de las Naciones Unidas (ONU), 2018, las mujeres predominan en el cuidado de las personas mayores en sus casas, lo que repercute en desigualdad de condiciones de trabajo, que las tiene en situación precaria. Países como España, cuentan con un subsidio para la ayuda a domicilio que puede ser la atención a necesidades básicas, de higiene y demás, limpieza del hogar y apoyo para actividades fuera del hogar. En otros países, se observan servicios que se pagan mediante presupuesto general, impuestos y primas (Turquía y Países Bajos). Singapur cuenta con un programa integral de envejecimiento, mientras en países del Caribe se han desarrollado programas de ayuda a domicilio, para aquellas personas mayores que no pueden vivir solas. Costa Rica, por ejemplo, con la Ley 7756, otorga licencia y subsidios a los responsables de cuidar a un enfermo en fase terminal, siendo en ocasiones la llamada familia solidaria.

Reconsiderando lo anterior, cabe señalar que la Oficina Nacional del Reino Unido, reportó que la mayoría de personas relacionaron como discriminación por vejez, el recibir un 
trato como mercancía en lugar de consumidores de derechos. En Australia, según Carpio \& Sar (2014), se informó sobre la vulnerabilidad económica, emanada de un largo periodo de desempleo antes de la edad jubilatoria, cosa que algunos países vincularon como discriminación por edad en el trabajo. En este aspecto, la Encuesta Nacional sobre Discriminación 2010, en México, arrojó que el 27.9\% de entrevistados consideraron que sus derechos no habían sido respetados por su edad. En Uganda, debido a la precaria economía que lleva a las personas mayores al abuso, estrés, pobreza crónica y abandono, aunado a esto el perder el trabajo, no se cuenta con la posibilidad de regresar a trabajar. Existen, además quienes demandan medidas especiales, como es el caso de las personas mayores migrantes de México (Organización Mundial de la Salud (OMS), 2010).

De acuerdo con el incremento en la esperanza de vida, en poco tiempo se contará con adultos mayores asistiendo a sus padres (Damián, y otros, 2019). Tal hecho concebirá carestía de índole física, económica, médica, por mencionar algunas, además de estar relacionadas con los Derechos Humanos, los cuales se deben vigilar de una manera estrecha a través de la elaboración o evaluación de las políticas vigentes. Los adultos mayores prefieren vivir en su casa y necesitan cuidadores, ya sea formales o informales (De Valle, Hernández, Zúñiga, \& Martínez, 2015), cuidadores que sean de su familia o gente cercana a ellos. De acuerdo con esto, se debe apoyar a las personas que desempeñan este rol, por parte de servicios sociales del ayuntamiento local, grupos de apoyo, voluntariado, asociaciones e iglesia (García, 2005).

Huenchuan (2002) indica que es así como, en el Reino Unido, se cuenta con Programas de Respiro, que ayuda con reemplazos para los cuidadores. En Irlanda, el Departamento de Protección Social entrega periódicamente un subsidio que puede ser usado para pagar el relevo en caso de necesitarlo; en países del Caribe, los centros de atención diurna se encargan de las personas mayores, para ayudar a las personas que trabajan, dado que alternativas como entrar a una residencia para estas situaciones de respiro, estresan mucho a los adultos mayores. Huenchuan (2002) alude por lo que se debe evaluar, previamente, en ciertas épocas del año, como en el verano en España que puede haber hasta tres millones de personas abandonadas por el motivo de las vacaciones de los familiares. Mismo autor menciona que en países como Estados Unidos, Canadá, Reino Unido, un porcentaje, que llega a ser 38\%, dedican más de 30 horas semanales al cuidado de personas con alguna enfermedad crónica o discapacidad. En México, 53 horas semanales; en Costa Rica, 50.8 horas semanales; en Argentina y Perú, 40 horas semanales. En países como México, una buena parte de cuidadores no remunerados suele ser la pareja u otra persona mayor. Se estima que, en algunos países, por cada 100 personas de 65 años o más, por ejemplo, en Australia, 4,4 son cuidadores formales y 83,3 informales; mientras, en Estados Unidos, llega a 6.4 formales, por 123 informales. Lo que hará que, en 2030, de acuerdo con la tendencia, el 50\% de la población económicamente activa, menor a 65 años, deberá dedicar el doble de tiempo que en 2015.

Se comprende que toda persona mayor debería estar protegida del maltrato como una forma de tortura. El Movimiento Internacional contra todas las Formas de Discriminación y Racismo (International Movement Against All Forms Of Discrimination and Racism (IMADR), 2011), en este contexto, y el Comité contra la Discriminación de las Mujeres, así como las Naciones Unidas, hace, en su recomendación No. 27, un Ilamado a que se tenga en cuenta el maltrato contra mujeres mayores. Esto, referente a violencia doméstica, física o sexual, particularmente si la mujer sufre de discapacidad. También tratado por la Convención Interamericana en los Artículos 9 y 10, que va más allá y plantea que se establezca legislación para la protección de los Derechos Humanos de las personas mayores, ya que, de acuerdo con la Organización Mundial de la Salud (OMS), 2018, 10\% de las personas mayores sufre algún tipo de maltrato ya mencionado. Investigaciones revelan que este tipo de maltrato produce mayor número de muertes que en aquellos 
Bañuelos et al - Desigualdad en los Derechos Humanos de las mujeres mayores: Caso Estado de Zacatecas.

casos en que no son maltratados, por lo que, en países como Estados Unidos, Austria, Canadá, Alemania, Reino Unido, Nueva Zelandia, ente otros, se pone mayor atención contra estas prácticas, con comités, protocolos y programas de prevención. Tenemos, pues, que en América Latina, países como Ecuador, en su Código Penal desde 2014, tiene penas de 3 a 19 años de cárcel por este tipo de violencia; en Uruguay, se cuenta con un Instituto Nacional de Personas Mayores; en República Dominicana, el Consejo Nacional de la Persona Envejeciente, en Bolivia y Argentina se cuenta también con leyes de prevención; en Trinidad y Tobago se ha implementado un servicio de ayuda a personas mayores; asimismo, en Chile; en México existe el Programa Nacional de Formación de Cuidadores de Adultos Mayores, capacitados por el Instituto Mexicano de Seguro Social. Además, se cuenta con programas como el Plan Geriátrico Institucional y el Programa de Envejecimiento Activo, con el propósito de prolongar la independencia física y mental de las personas mayores (Secretaría de Salud (SS), 2006).

Respecto al cuidado a largo plazo, el rol de la mujer y el aumento de la sobrevivencia con enfermedades, en América Latina el 26.1\%, de la población, entre niños y personas mayores de 75 años, necesitaban cuidados especiales; son personas que necesitan atención por caídas, medicamentos y padecen enfermedades crónicas, en la que la familia ya no apoya como en tiempos pasados recientes. Facilidades como horario flexible, permisos, asesoría, capacitación, incluso un salario ciudadano, ayudaría a afrontar esta situación. Adaptar políticas de apoyo a familiares que brindan atención domiciliaria a personas mayores, mejorando los sistemas tradicionales, otorgando gratificación, materiales y otros para aquellos que cuidan personas mayores (Watch, 2012), podría contribuir de forma positiva.

Dado que, en la presente investigación, para el caso del contexto estudiado, el propósito consistió en investigar si las personas mayores sufren desigualdad de sus Derechos humanos, particularmente las mujeres en el estado de Zacatecas, México, se consideraron los Derechos Humanos Universales, mismos que corresponden con el objeto de estudio y que se encuentran mencionados en la Tabla 1. Destacan, por el contenido, aquellos que se refieren o tienen que ver con la igualdad que debe de haber entre los seres humanos, entre ellos; que los seres humanos nacen libres e iguales en dignidad y derechos, sin discriminación o tratos crueles, inhumanos o degradantes; ya sea por raza, sexo o religión, con un derecho a remuneración equitativo que asegure alimentación, vestido, vivienda, asistencia médica, educación (Organización de las Naciones Unidas-ONU, 2014).

Tabla 1. Derechos Humanos Universales.

\begin{tabular}{|c|c|}
\hline Artículo & Menciona o trata sobre: \\
\hline 1. & Todos los seres humanos nacen libres e iguales en dignidad y derechos \\
\hline 2. & Sin distinción de raza, color, sexo, religión \\
\hline 3. & Derecho a la vida, a la libertad y a la seguridad de su persona \\
\hline 5. & A no sufrir tratos crueles, inhumanos o degradantes. \\
\hline 7. & A la no discriminación \\
\hline 22. & Seguridad Social; satisfacción de los derechos económicos, sociales, y culturales. \\
\hline 23.3 & $\begin{array}{l}\text { Derecho a remuneración equitativa y satisfactoria, que asegure la dignidad humana } \\
\text { Y que se deberá completar, en caso de ser necesario, con programas sociales }\end{array}$ \\
\hline 25 & $\begin{array}{l}\text { Derecho a un nivel de vida adecuado, que incluya alimentación, vestido, vivienda, asistencia médica y servicios sociales, } \\
\text { así como seguro en invalidez, viudez y vejez }\end{array}$ \\
\hline 26 & Derecho a la Educación \\
\hline
\end{tabular}

Nota. Fuente: (Organizacion de las Naciones Unidas (ONU), 2018). 
Para estar al tanto, de si existía desigualdad de los Derechos Humanos Universales de las mujeres mayores, mencionados anteriormente, para el caso del Estado de Zacatecas se procedió a realizar una investigación empírica mediante la aplicación de un cuestionario, elaborado conjuntamente con: la Comisión estatal de los Derechos Humanos de Zacatecas, la Unidad Académica de Contaduría y Administración y Medicina Humana, estas últimas instituciones pertenecientes a la Universidad Autónoma de Zacatecas, México; dicho instrumento estuvo avalado por investigadores expertos de ésta misma Universidad, en la que se recogieron datos referentes, que permitieran contrastar las condiciones en que viven las mujeres mayores de nuestra entidad.

Objetivo de Investigación: El objetivo del presente estudio consistió en evaluar y determinar la desigualdad de los Derechos Humanos que viven las mujeres mayores, en el Estado de Zacatecas, México.

\section{METODOLOGÍA}

Tipo de estudio. El presente estudio tuvo un enfoque cuantitativo en el nivel investigativo explicativo, en un contexto observacional y prospectivo, ya que se obtuvieron datos a propósito de la investigación (primarios); transversal, puesto que se midió una sola ocasión durante los meses de noviembre y diciembre de 2018.

Población y Muestra. El estudio se llevó a cabo en Zacatecas, estado de la República Mexicana con una población de 1'579,209 habitantes (Instituto Nacional de estadística y Geografía (INEGI), 2017). La muestra consistió en 2.566 personas mayores de 65 años dentro de las ocho zonas más representativas de los municipios del estado que reconoce la Comisión estatal de derechos Humanos (CEDH), 2016.

Instrumento. Para la presente investigación, se diseñó un instrumento compuesto por 77 preguntas de las cuales 25 estuvieron en escala de Likert midiendo apoyo familiar, maltrato, abuso, dependencia y alimentación por medio de las opciones $1=$ Nunca, 2= Casi nunca, 3= Casi siempre y 4= Siempre; además de los apartados como: datos generales que contiene 14 preguntas, entre ella: municipio, localidad, género, estado civil, con quien vive, dependientes económicos, entre otros; cinco preguntas que contienen: antecedentes sobre migración, nueve se refieren a nivel socioeconómico, 10 referente a ocupación e ingreso, una además de servicios médicos, cuatro de asistencia social, dos sobre discapacidad, dos sobre el cuidado del adulto mayor; las cinco restantes contestadas por el entrevistador, referentes a la higiene, así como su temor y estado de ánimo del entrevistado.

Procedimiento: Se aplicó la encuesta cara a cara, mediante el procedimiento de acudir a las comunidades previamente seleccionadas, con las personas mayores, quienes fueron las unidades de análisis (Tabla 2), se solicitó la información, haciéndoles saber de la confidencialidad de los datos.

Tabla 2. Ficha Técnica de la Investigación.

\begin{tabular}{ll}
\hline \multicolumn{1}{c}{ Características } & \multicolumn{1}{c}{ Encuesta } \\
\hline Universo & $\begin{array}{l}\text { Personas mayores de } 65 \text { años del Estado de Zacatecas, México. } \\
\text { Ocho regiones del Estado de Zacatecas de acuerdo a la Comisión Estatal de Derechos Humanos } \\
\text { del estado de Zacatecas (CEDHEZ). }\end{array}$ \\
Ámbito de estudio & $\begin{array}{l}\text { Persona mayor a } 65 \text { años } \\
\text { Unidad de análisis }\end{array}$ \\
$\begin{array}{l}\text { Método de recogida de información } \\
\text { Procedimiento de muestreo }\end{array}$ & Entrevista personal a las personas mayores seleccionadas \\
Tamaño de la muestra & 2566 \\
Margen de error del muestreo & $5 \%$ a un nivel global para una confianza de $95 \% \mathrm{p}=\mathrm{q}=0.5$ \\
Fecha de trabajo de campo & Noviembre -Diciembre de 2018 \\
\hline
\end{tabular}

Nota. Fuente: Elaboración Propia. 
Bañuelos et al - Desigualdad en los Derechos Humanos de las mujeres mayores: Caso Estado de Zacatecas.

\section{RESULTADOS}

Entre los estadísticos descriptivos, predomina: del total de la muestra un $50.3 \%$ son hombres y el resto $49.7 \%$ mujeres; el $67.3 \%$ de los entrevistados vive en el sector rural, el resto en el urbano. Además, prevalecen las mujeres en el rango de edad de entre 65 a 75 años. A mayor edad, estas, son menos que los hombres; viven menos en pareja, por lo que se determinó que son cuidadas por familiares y conocidos. Sin embargo, no están satisfechas con la ayuda que les brindan, se sienten solas. En su mayoría no trabajan; su ingreso depende mayormente de sus familiares. Esto, a pesar de estar en un programa de gobierno. Perciben menos de 560 pesos semanales; en su mayoría tienen alguna discapacidad para moverse, ver u oír, entre otras, y se han quedado sin comer algunas veces. Las han hecho sentir temor, vergüenza; en algunos casos han sido objeto de abusos físicos y de confianza. Al momento de la entrevista se observaron sucias, tristes, deprimidas o asustadas.

En el resultado del análisis factorial exploratorio, se encontraron tres factores, que explican en conjunto el $69.72 \%$ de la varianza total explicada (Hair, Black, Babin, \& Anderson, 2010), como se puede apreciar en la Figura 1. El primero de ellos, que agrupa cinco ítems, tienen que ver con estar satisfecho con la familia, respecto a: ayuda, tiempo que se les dedica, conversar sobre los problemas, tomar decisiones en conjunto; de acuerdo con la naturaleza de estos se nombró: apoyo familiar; el segundo factor, que agrupa a cuatro ítems, referentes a: si les han gritado sin justificación, hacerlos sentir tristes, avergonzados, temerosos, ansiosos, infelices, se hayan aprovechado, abusado o tratado de usar su dinero; este factor se nombró: abuso físico, psicológico y económico; el último, que agrupa dos ítems, relacionados con: dependencia para tareas básicas como bañarse, vestirse, ir al baño o comer, ir a las compras y pagar servicios; este mencionado factor, se nombró: dependencia física y de servicios.

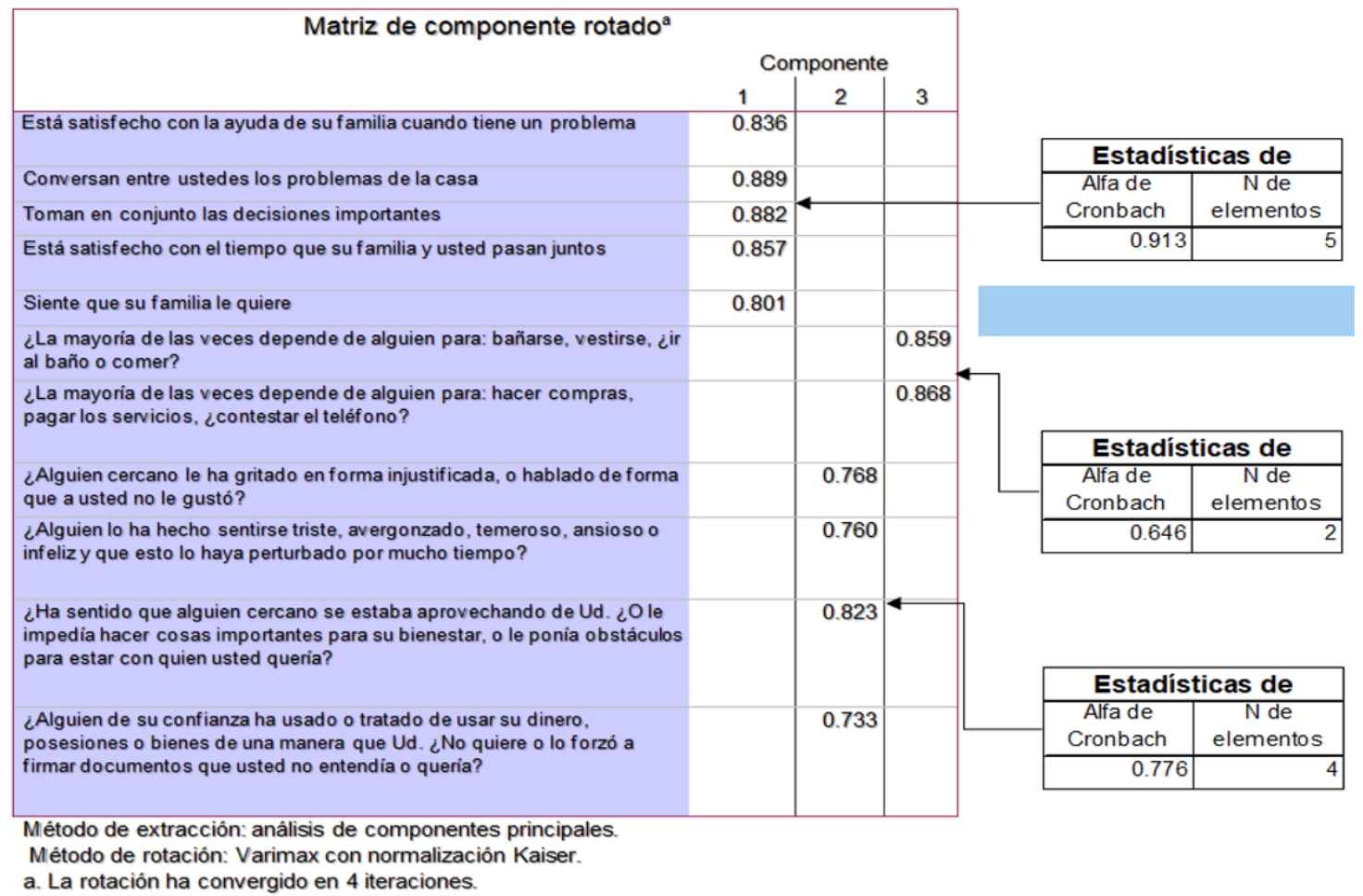

Figura 1. Matriz de Componente Rotado.

Fuente: Paquete estadístico SPSS versión 21. 
Una vez encontrados los factores que agrupan la percepción que sobre sus derechos tienen, se obtuvo la frecuencia de estos, como se muestra en la figura 2.

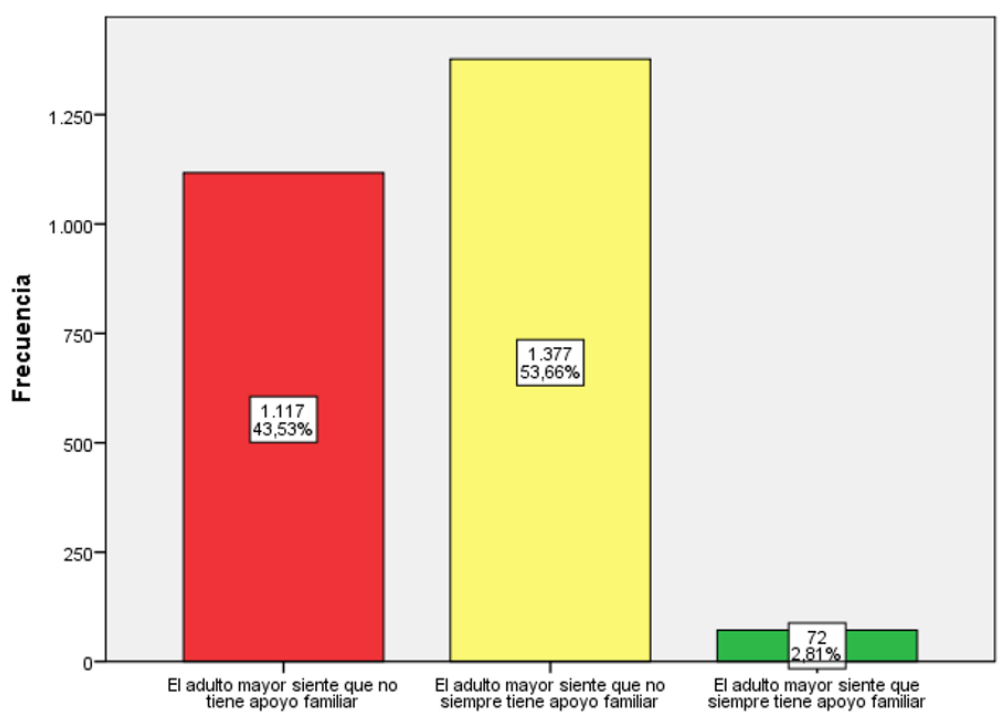

Figura 2. Apoyo familiar al adulto mayor. Fuente: Paquete estadístico SPSS versión 21.

De acuerdo con la Figura 2. Referente al primer factor: apoyo familiar, se encontró que la percepción del adulto mayor en un $45.53 \%$, no cuenta con este. Respecto a los problemas, la toma de decisiones importantes, así como al tiempo que les dedican y a sentirse queridos, un $53.66 \%$, percibe que no siempre tiene apoyo familiar. Por consiguiente, solamente un $2.81 \%$ se siente apoyado todo el tiempo.

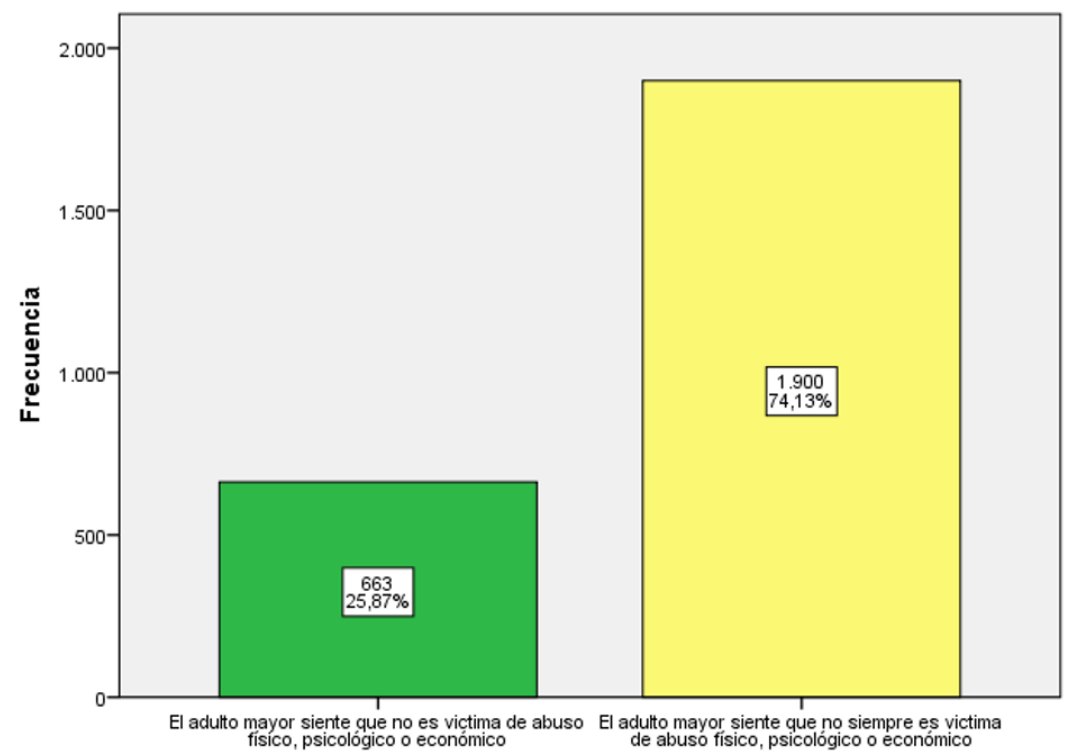

Figura 3. Abuso físico, psicológico y económico contra el adulto mayor. Fuente: Paquete estadístico SPSS versión 21.

La Figura 3, que hace referencia al segundo factor: abuso físico, psicológico y económico, que contempla que haya sido violentado con gritos, le hagan sentir vergüenza, temor, 
ansia, infelicidad, así como que le impidan hacer algo a favor de su bienestar, como lo sería usar su dinero, firmar documentos sin su consentimiento, el adulto mayor percibió en casi $26 \%$, que no es víctima dentro de este factor. Sin embargo, se encontró un $74.13 \%$ que afirma que no siempre lo es, por lo que es preocupante, puesto que conlleva haber tenido, por lo menos, un evento de esta naturaleza de abuso.

En cuanto al tercer factor, como se observa en la Figura 4, denominado: Dependencia física y servicios, contempla que la mayoría de las veces el adulto mayor depende de alguien para bañarse, vestirse, ir al baño o comer, hacer las compras, pagar servicios o contestar el teléfono, el $14.96 \%$ de los encuestados contempla que siente que no depende de otros para realizar actividades físicas o de servicios; un $85.04 \%$, siente que no siempre depende de otros.

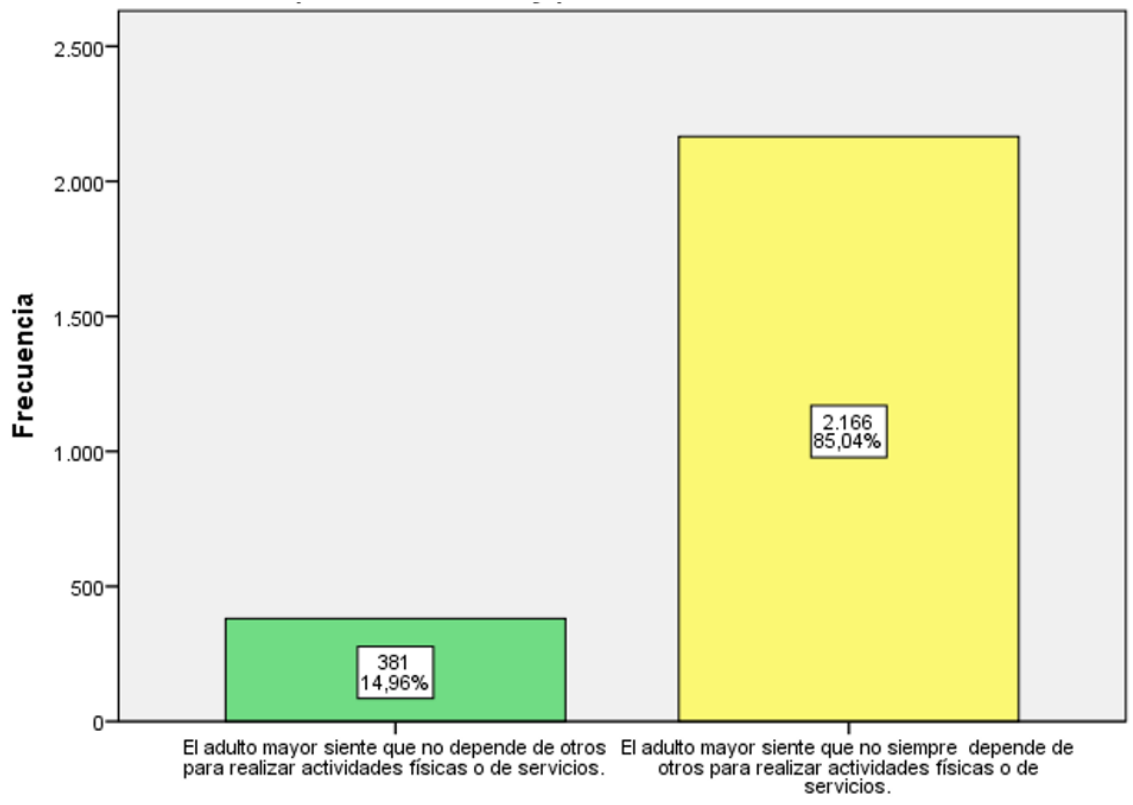

Figura 4. Dependencia física y servicios. Fuente: Paquete estadístico SPSS versión 21.

Tablas Cruzadas usando Chi Cuadrado de Pearson. Usando la prueba de Chi Cuadrada de Pearson, para buscar relación entre variables, en este caso, se utilizó para encontrar relaciones estadísticamente significativas entre las características de las mujeres en la muestra y una serie de ítems relacionados con el abuso de sus derechos humanos, como lo muestra la Tabla 3.

De la Tabla 3, para fines de la investigación, cabe destacar que está relacionado el ser mujer con las siguientes variables como lo son: vivir en el medio rural, vivir más que el hombre en el rango de 65-75, pero menos que ellos, para las personas mayores de 85 años, ser remunerada con $\$ 560$ pesos (aquellas que tienen ingresos), además muy pocas remuneradas con cantidades mayores a la mencionada, tienen como fuente de ingreso principal el apoyo familiar, se encontraron sin pareja, aunado a que tienen dificultades para oír, ver, usar brazos y manos, así como usar bastón entre otras, las personas mayores dependen de alguien para asearse, realizar compras de alimentos y medicamentos, perciben malos tratos como gritos, sentimientos de tristeza, entre otros. 
Tabla 3. Resultado de Pruebas de Hipótesis de Chi Cuadrado de Pearson

\begin{tabular}{|c|c|c|}
\hline Variable & Sig. & Interpretación \\
\hline Rural & 0.000 & Mayormente es la mujer la que vive en el medio rural \\
\hline Edad (rango de 86 años o más) & 0.005 & $\begin{array}{l}\text { En el rango de edad de } 65-75 \text { años, el número de mujeres supera al de los hombres, } \\
\text { pero en el rango de edad de } 86 \text { años o más, el número de hombres supera al de las } \\
\text { mujeres, }\end{array}$ \\
\hline $\begin{array}{l}\text { A cuánto asciende su ingreso semanal } \\
\text { en pesos }\end{array}$ & 0.000 & $\begin{array}{l}\text { En cuanto al ingreso menor o igual a } \$ 560 \text {, la proporción de mujeres que participan } \\
\text { en esta categoría es mayor que la de los hombres, cuanto más se eleva el ingreso, la } \\
\text { proporción de mujeres disminuye respecto de la del hombre. }\end{array}$ \\
\hline $\begin{array}{l}\text { Ppal. Fuente de ingreso: apoyo familiar, } \\
\text { jubilación, programas }\end{array}$ & 0.000 & $\begin{array}{l}\text { La principal fuente de ingreso de las mujeres es el apoyo familiar. Respecto al apoyo } \\
\text { de programas sociales, PROSPERA apoya más a la mujer en proporción al hombre, } \\
\text { cosa contraria ocurre del programa INAPAM. }\end{array}$ \\
\hline $\begin{array}{l}\text { Estado civil, vivir con el cónyuge o } \\
\text { pareja, hijos, nietos. } \\
\text { Ser cuidada por hijos, nietos, vecinos o } \\
\text { nuera. }\end{array}$ & 0.000 & $\begin{array}{l}\text { Predomina que la mujer se encuentra sola, sea por soltería, divorcio o viudez; } \\
\text { propensa a vivir con sus hijos o nietos y que quien cuide de ellas sea ellos mismos, } \\
\text { los vecinos o la nuera; mientras que el hombre viva con su pareja y sea ella quien lo } \\
\text { cuide. }\end{array}$ \\
\hline $\begin{array}{l}\text { Tiene dificultad para moverse o } \\
\text { caminar, ver, oír, usar bastón, andadera, } \\
\text { dificultades en brazos o manos. }\end{array}$ & 0.001 & $\begin{array}{l}\text { Es mayor la proporción de mujeres que padecen dificultades para ver, para oír y para } \\
\text { usar sus brazos o manos, y que necesite el apoyo de lentes, bastón y andadera, caso } \\
\text { contrario ocurre con los audífonos, que predomina el hombre. }\end{array}$ \\
\hline $\begin{array}{l}\text { La mayoría de las veces depende de } \\
\text { alguien para bañarse, hacer compras, } \\
\text { pagar servicios. }\end{array}$ & 0.000 & $\begin{array}{l}\text { Si bien la mayoría de los encuestados no se identifican con estas variables, la } \\
\text { proporción de mujeres que sí dependen de alguien para bañarse, hacer compras, } \\
\text { pagar servicios, etcétera es mayor que la de hombres. }\end{array}$ \\
\hline $\begin{array}{l}\text { Alguien impidió que usted obtuviera } \\
\text { comida, ropa, medicamento. Alguien } \\
\text { cercano le grito }\end{array}$ & 0.029 & $\begin{array}{l}\text { La proporción de mujeres que sí sienten que alguien les impidió obtener comida o } \\
\text { medicamentos o que sienten que alguien les grito es mayor que la de hombres. }\end{array}$ \\
\hline $\begin{array}{l}\text { Cuenta con apoyo familiar económico, } \\
\text { emocional, asistencial, material, solución } \\
\text { de problemas. Se siente querido }\end{array}$ & 0.000 & $\begin{array}{l}\text { Esta relación de variables nos indica que, en comparación con el hombre, es la } \\
\text { mujer la que más cuenta con el apoyo familiar, sobre todo en el aspecto económico, } \\
\text { emocional, asistencial y material. Situación que es reforzada con el hecho de que sea } \\
\text { la proporción de mujeres mayor que la de los hombres, respecto de sentir satisfacción } \\
\text { con el tiempo que pasa por su familia y la ayuda que esta le brinda; tiene problemas, } \\
\text { conversar los problemas en casa y sentir que su familia le quiere. }\end{array}$ \\
\hline $\begin{array}{l}\text { Edo. De ánimo, preocupación, } \\
\text { sentir vergüenza, soledad, temor, } \\
\text { incomodidad. }\end{array}$ & 0.000 & $\begin{array}{l}\text { Aunque la mayoría de la muestra no se reveló temerosa, para contestar y manifiestan } \\
\text { un estado de ánimo tranquilo, la proporción de mujeres tristes, es mayor que la de } \\
\text { hombres, pero menor, sentirse, avergonzadas, enojadas o solas. }\end{array}$ \\
\hline $\begin{array}{l}\text { El espacio donde se encuentra el adulto } \\
\text { se ve cómodo, limpio y bien atendido. }\end{array}$ & 0.000 & $\begin{array}{l}\text { La relación entre estas variables indica que la proporción de mujeres que se encuentran } \\
\text { en espacio cómodo y limpio es mayor que la proporción de los hombres; y que la } \\
\text { proporción de hombres que se ven desaliñados, sucios y mal atendidos es mayor que } \\
\text { la de las mujeres. }\end{array}$ \\
\hline
\end{tabular}

Nota. Fuente: Paquete estadístico SPSS versión 21.

\section{DISCUSIÓN}

La Declaración Universal de los Derechos Humanos, expresa sobre los derechos y libertades a que somos meritorios los seres humanos, de manera exclusiva y en condiciones de igualdad. Todas las personas tienen derechos económicos, sociales, culturales y cívicos, entre otros, entre los resultados encontrados en la presente investigación, se destaca que un $74 \%$ de los entrevistados consideró que en ocasiones han sido objeto de abuso físico, psicológico, o económico, siendo mayor que el $27.9 \%$ que arrojó la Encuesta Nacional sobre Discriminación 2010 en México, en la que los entrevistados consideraron que sus derechos no habían sido respetados por su edad, mientras que la Organización Mundial de la Salud menciona que el $10 \%$ ha estado en esta misma situación (Organización Mundial de la Salud (OMS), 2010). A partir que se celebró en México la primer Conferencia Mundial sobre la Mujer los gobiernos del mundo discuten 
Bañuelos et al - Desigualdad en los Derechos Humanos de las mujeres mayores: Caso Estado de Zacatecas.

sobre la problemática de la mujer y exhortan a realizar gestiones concretas con el fin de abatir la discriminación en diferentes aspectos de la vida de éstas. Las Naciones Unidas, en su nota conceptual relativa al proyecto de recomendación general sobre la mujer de edad y la protección de sus Derechos Humanos, aprobó una recomendación sobre los Derechos Humanos de la mujer de la tercera edad, para evitar discriminación y tener condiciones de igualdad, De acuerdo con la prueba de Chi Cuadrado, se encontró que está relacionado el ser mujer y una serie de situaciones, entre ellas, que estas sufren de abandono, malos tratos, carencia económica y psicológica, además de que no tienen trabajo remunerado, lo que confirma el apoyo familiar como fuente principal de ingreso, que es de menos de $\$ 560$ pesos semanales, violación a los preceptos enunciados en la Declaración de los Derechos Humanos Universales (Organización de las Naciones Unidas-ONU, 2014).

Otros hallazgos en la presente investigación, hacen referencia a que la mujer mayor sufre de una serie de padecimientos como lo son: el no ver y escuchar bien; algún tipo de problema en el uso de sus extremidades la hace necesitar más ayuda en razón que el hombre; además de que las mujeres se sintieron alguna vez, limitadas para la obtención de sus necesidades básicas como comida o medicamentos, al mismo tiempo tuvieron la sensación de tristeza; por otra parte, aunque en porcentajes bajos, se encontró el maltrato, abuso físico y psicológico en las mujeres encuestadas; preocupadas por dinero, aun cuando cuentan con apoyos de programas asistenciales que les otorgan, ellas perciben que es insuficiente.

En el contexto en que se desarrolló esta investigación, se encontró una frecuencia más alta en las mujeres que en los hombres, en el rango de edad de 65 a 75 años, con $63.7 \%$ y $57.6 \%$ respectivamente, caso contrario a los rangos de 76 a 85 y mayores de 86 años, en el que hombres predominan, $5 \%$ y $1 \%$ respectivamente en los mencionados rangos, esto a diferencia del resto de la República Mexicana, en donde las mujeres viven en promedio más años que los hombres; la esperanza de vida en 2016 se ubicó en casi 78 años para las mujeres y cerca de 73 años para los hombres (Instituto Nacional de estadística y Geografía (INEGI), 2017). Incluso del mundo, en donde las mujeres alcanzan 75.3 años y para los hombres 69.8 años, de acuerdo con la Organización de las Naciones Unidas-ONU (2014).

A pesar de las resoluciones, declaraciones y recomendaciones aprobadas Las Naciones Unidas, las mujeres siguen siendo objeto de importantes exclusiones, violando con esto la igualdad de sus derechos, por lo que se condena la discriminación en todas sus formas. Toda persona mayor debería estar protegida del maltrato como una forma de tortura. Es necesario contribuir con investigaciones empíricas, que den sustento a políticas públicas en las que se tomen en cuenta todos los aspectos señalados como Derechos Humanos de las personas mayores. Esto, sin descuidar la equidad de género.

\section{REFERENCIAS}

Carpio, M. E., Sar, S. O. (2014). Alcances del Principio de Igualdad. Universidad de San Martín de Porres.

Carrera, L. E. (01 de 10 de 2019). https://www.segobver.gob.mx/genero/docs/Biblioteca/ Los derechos\%20humanos 
Comisión estatal de derechos Humanos (CEDH). (2016). Programa Estatal de Derechos Humanos. Comisión Nacional de Derechos Humanos Zacatecas.

Comisión Nacional de Derechos Humanos (CNDH). (2018). Comisión Nacional de Derechos Humanos. Gaceta, 194.

Consejo Nacional de Población (CONAPO). (07 de 2019). https://wwhttps://www.gob. $\mathrm{mx} / \mathrm{cms} / \mathrm{uploads} / \mathrm{attachment/file/487365/32 \text {ZAC.pdf }}$

Damián, J., Pastor, B., García, L., Ruigómez, A., Martínez, M. P., de Pedro, C. (2019). Facility ownership and mortality among older adults residing in care homes. PLoS ONE, https://doi.org/10.1371/journal.pone

De Valle, A., Hernández, L., Zúñiga, V. M., Martínez, A. (2015). Sobrecarga y Burnout en cuidadores informales del adulto mayor. Enfermería universitaria, 12(1), 19-27.

García, V. (2005). Enciclopedia de la salud. 2005. Apoyo a los cuidadores de las personas mayores dependientes. https://mx.hola.com/salud/enciclopediasalud/2005060945407/mayores/generales/apoyo-a-los-cuidadores-de-laspersonas-mayores-dependientes/

Hair, J. F., Black, W. C., Babin, B. J., Anderson, R. E. (2010). Multivariate data analysis (7th ed.). Prentice Hall.

Huenchuan, S. (2002). Envejecimiento, personas mayores y agenda 2030 para el desarrollo sostenible. Santiago: CEPAL. Obtenido de Arias A. 2002. España abandona a sus mayores. ABC.es. España Recuperado en 15 de Febrero de 2019. https://www. abc.es/hemeroteca/historico-30-07-2002/abc/Sociedad/espa\%C3\%B1a-abandonaa-sus-mayores 117821.html

Instituto Latinoamericano de las Naciones Unidas para la Prevención del Delito y Tratamiento del Delincuente (ILANUD). (2001). Declaración Universal de Derechos Humanos: Texto y Comentarios. Programa Mujer, Justicia y Género.

Instituto Nacional de estadística y Geografía (INEGI). (2017). Mujeres y Hombres en México 2017. Aguascalientes, Aguascalientes, México. http://cuentame.inegi.org. $\underline{\mathrm{mx} / \text { poblacion/esperanza.aspx?tema }=\mathrm{P}}$

International Movement Against All Forms Of Discrimination and Racism (IMADR). (2011). La Convención Internacional sobre toda forma de discriminación racial (ICERD) y su Comité (CERD). IMADR.

Organización de las Naciones Unidas (ONU). (2014). Los derechos de la mujer son derechos humanos. Organización de las Naciones Unidas.

Organización de las Naciones Unidas (ONU). (2018). Informe de los objetivos de Desarrollo Sostenible. Naciones Unidas.

Organización Mundial de la Salud (OMS). (2010). Salud mental y desarrollo: poniendo a las personas con problemas de salud mental como un grupo vulnerable. Organizacion Mundial de la Salud. 
Bañuelos et al - Desigualdad en los Derechos Humanos de las mujeres mayores: Caso Estado de Zacatecas.

Organización Mundial de la Salud (OMS). (2015). Informe Mundial sobre el Envejecimiento y la Salud. Organización de las Naciones Unidas.

Sánchez, V. R. (2018). Derechos Humanos, Seguridad Humana, Igualdad y Equidad de Género. Comisión Nacional de los Derechos Humanos.

Secretaría de Salud (SS). (2006). Informe Nacional Sobre Violencia y Salud. Secretaría de Salud.

Watch, T. F. (2012). La familia como agente de la salud. Instituto Internacional de estudios sobre la Familia. 